\section{ORIGINAL RESEARCH}

K. Lin

K.S. Kazmi

M. Law

J. Babb

N. Peccerelli

B.K. Pramanik

\title{
Measuring Elevated Microvascular Permeability and Predicting Hemorrhagic Transformation in Acute Ischemic Stroke Using First-Pass Dynamic Perfusion CT Imaging
}

\begin{abstract}
BACKGROUND AND PURPOSE: Hemorrhagic transformation (HT) can be a devastating complication of acute ischemic stroke (AIS). The purpose of this study was to determine whether increased microvascular permeability (PS) of the blood-brain barrier was detected in early AIS by using first-pass dynamic perfusion CT (PCT) and whether PS was significantly higher in infarcts destined for HT.

MATERIALS AND METHODS: Fifty patients with AIS less than 3 hours old and evaluated by PCT were included. PS color maps were retrospectively generated from PCT data using the Patlak model. One reader analyzed each PS map by drawing 4 circular $10-\mathrm{mm}$ regions of interest on any focal abnormality.

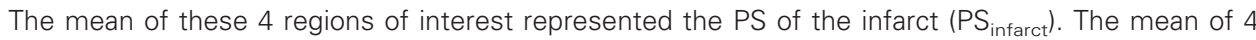
mirror regions of interest on the nonischemic contralateral hemisphere was also obtained ( $\left.P S_{\text {control }}\right)$. $\mathrm{PS}_{\text {infarct }}$ and $\mathrm{PS}_{\text {control }}$ were compared by using an exact Wilcoxon test. $\mathrm{PS}_{\text {infarct }}$ for infarcts that developed HT on follow-up ( $\mathrm{PS}_{\mathrm{HT}}$ ) was compared with all of the others $\left(\mathrm{PS}_{\mathrm{No}-\mathrm{HT}}\right.$ ) using an exact Mann-Whitney test.
\end{abstract}

RESULTS: Forty-four infarcts (88\%) showed focal PS elevation in the region of infarct. In units of milliliters per 100 milliliters per minute, PS $_{\text {infarct }}$ ranged from 0 to 13 (mean: $3.5 \pm 3.1$ ) versus PS $_{\text {control }}$ of $0-0.8$ (mean: $0.28 \pm 0.27 ; P<.0001$ ). Six infarcts $(12 \%)$ developed $\mathrm{HT}$, all of which were within the region of PS elevation. $\mathrm{PS}_{\mathrm{HT}}$ ranged from 5.2 to 13 (mean: $9.8 \pm 2.9$ ) versus $\mathrm{PS}_{\mathrm{No}-\mathrm{HT}}$ of $0-5.9$ (mean: $2.7 \pm 2.0 ; P<.0001)$. Eighteen infarcts (36\%) were treated with recombinant tissue plasminogen activator (rtPA). A significant difference between $\mathrm{PS}_{\mathrm{HT}}$ and $\mathrm{PS}_{\mathrm{No}-\mathrm{HT}}$ persisted irrespective of rtPA treatment.

CONCLUSIONS: Elevated permeability was detectable in AIS by using first-pass PCT and it predicted subsequent $\mathrm{HT}$.

B olus-chase perfusion imaging ${ }^{1-10}$ uses dynamic contrastenhanced data to produce parametric color overlay maps of cerebral blood flow (CBF), cerebral blood volume (CBV), and mean transit time or time of local peak enhancement (TTP). These standard perfusion metrics are used to detect an acute ischemic stroke (AIS) and the presence of "penumbra," the hypoperfused but potentially salvageable tissue at risk of infarction. In various acute care centers, dynamic perfusion CT (PCT) is one component of a multimodal "CT stroke series," which also consists of a noncontrast CT (NCCT) of the brain to exclude intracerebral hemorrhage and other stroke mimickers and a CT angiogram (CTA) of the cervical and intracranial arteries to identify the site of vessel stenosis or occlusion. If such a comprehensive CT protocol can yield additional information regarding the risk of hemorrhagic transformation (HT), it will further help the selection of candidates for thrombolytic therapy.

HT is generally thought to arise from ischemic damage to the blood-brain barrier (BBB) followed by reperfusion either through recanalization of the occluded vessel or through col-

Received August 1, 2006; accepted after revision November 21.

From the Departments of Radiology (K.L., K.S.K., M.L., J.B., N.P., B.K.P.) and Neurosurgery (M.L.), New York University Medical Center, New York, NY.

Paper previously presented at: Annual Meeting of the American Society of Neuroradiology, May 2, 2006; San Diego, Calif.

Address correspondence to Meng Law, MD, Associate Professor of Radiology and Neurosurgery, Mount Sinai Medical Center, One Gustave L. Levy Place, New York, NY 10029; e-mail: meng.law@mountsinai.org

DOI 10.3174/ajnr.A0539 lateral circulation. ${ }^{11}$ Microvascular permeability (expressed as the transendothelial transfer constant $\left[\mathrm{k}^{\mathrm{PS}}\right]$ or permeability surface area product [PS]) is a metric of BBB integrity. Like the standard perfusion metrics, PS can also be calculated using dynamic imaging by measuring the leakage of an intravascular tracer into the extravascular (interstitial) space. ${ }^{12-15}$ In the normal brain parenchyma, PS is $\sim 0$ for relatively large hydrophilic molecules (such as a peripherally injected iodinated contrast agent), which reflect the tight regulation of the BBB. Multiple disease processes, however, including severe ischemia, can alter BBB integrity and allow the diffusion of fluid, blood, or contrast molecules into the interstitium. A nonzero PS represents this diffusion quantitatively, and its functional color map can be semiautomatically generated by dedicated commercially available software. Evaluating PS has already found tremendous use in tumor imaging, where elevated measurements secondary to neovascular alteration of the BBB are correlated to histologic grade. ${ }^{16-19}$

Can measurements of PS play a similarly important role in stroke imaging? Evidence from animal and human studies ${ }^{20-23}$ suggests that increased permeability can occur in the first 2-4 hours of acute ischemia. Kassner et $\mathrm{al}^{24}$ recently used dynamic MR permeability imaging to identify an association between HT and elevated permeability within 24 hours of ictus in a small series of 10 patients who were not treated with recombinant tissue plasminogen activator (rtPA). Rowley ${ }^{25}$ reported observations of focally elevated permeability in acute infarcts by using dynamic CT data, though the clinical significance of this elevation was not completely understood. 
The purpose of this retrospective study was to determine whether elevated microvascular PS can be detected in AIS using first-pass dynamic data acquired from standard PCT and whether there was a difference in PS between infarcts that developed HT and those that did not.

\section{Materials and Methods}

\section{Patient Demographics}

Imaging and clinical data obtained as part of standard clinical stroke care at our institution were retrospectively reviewed. Approval for this study was obtained from the Institutional Board of Research Associates, and the entire study was Health Insurance Portability and Accountability Act compliant. A retrospective waiver of consent was obtained from our institutional review board for review of all of the images and charts.

From January 2004 to April 2006, 85 consecutive patients presented to our institution emergency department within 3 hours from onset of symptoms that were suggestive of an acute hemispheric stroke (eg, hemiparesis, hemisensory loss, or aphasia) and had an NCCT, PCT, and CTA (CT stroke series) emergently performed. The patients in this study had neither been evaluated nor been treated at an outside facility before presentation. Laboratory tests and medical charts were reviewed to exclude the presence of acute renal insufficiency and iodinated contrast agent allergy. The decision to administer rtPA was made by the primary stroke management team after the evaluation of the CT stroke series.

Of the 85 consecutive patients, 50 (27 women and 23 men; aged 33-91 years) were included in our retrospective study for having had an acute nonlacunar $(>15 \mathrm{~mm})$ infarct detected on the PCT and confirmed on follow-up diffusion-weighted MR imaging (DWI) or serial CT examinations. Thirty-five patients were excluded for having either no evidence for AIS on initial or follow-up imaging and same day resolution of the presenting neurologic deficit (29 patients) or an acute infarct not within the spatial coverage of the section-selective PCT as determined by subsequent whole-brain DWI (6 patients).

\section{CT and MR Acquisition}

The NCCT was performed immediately before the PCT to evaluate for possible intracerebral hemorrhage and other stroke mimickers. All of the initial NCCTs, PCTs, and follow-up NCCTs were performed on a 16-section scanner (Sensation, Siemens, Erlangen, Germany). NCCT was obtained with 5-mm contiguous axial sections from vertex to skull base using imaging parameters of $120 \mathrm{kVp}, 400$ mAs, 1.5-mm section collimation, and 1-second rotation. PCT was obtained with 2 contiguous 12 -mm-thick axial sections centered at the level of the basal ganglia and internal capsule. A 60-second cine series was performed beginning 4 seconds after the intravenous administration of $50 \mathrm{~mL}$ of iodinated contrast at $4-5 \mathrm{~mL} / \mathrm{s}$ by a power injector into an antecubital vein (Omnipaque, $300 \mathrm{mg}$ of iodine/mL; GE Healthcare, Piscataway, NJ). PCT imaging parameters were 80 $\mathrm{kVp}, 200 \mathrm{mAs}, 0.5$-second rotation, and 60 images per section.

All of the follow-up MR examinations were performed on 1.5T systems (Vision or Symphony; Siemens). A localizing sagittal T1weighted image was obtained followed by nonenhanced axial T1weighted spin-echo (TR: $600 \mathrm{~ms}$; TE: $14 \mathrm{~ms}$ ), axial fluid-attenuated inversion recovery (TR: $9000 \mathrm{~ms}$; TE: $110 \mathrm{~ms}$; inversion time: 2500 ms), T2-weighted (TR: $3400 \mathrm{~ms}$; TE: $119 \mathrm{~ms}$ ), and diffusion-weighted (TR: 3400 ms; TE: 95 ms; B values 0, 100, and 1000) images.

\section{Data Processing}

At the time of acquisition, each patient's PCT dynamic images (dataset) were recorded on magnetic optical disks (MODs). In our retrospective study, these raw datasets were loaded from the MODs onto an off-line workstation (Leonardo; Siemens) equipped with a postprocessing software package to generate color overlay maps of dynamic cerebral enhancement data (Syngo Neuro Perfusion CT; Siemens). This application offers 2 different evaluation modes. The "standard perfusion" mode calculates the perfusion metrics of CBF, $\mathrm{CBV}$, and TTP for AIS evaluation by using the maximal slope method as described previously. ${ }^{2-4} \mathrm{CBF}$ is calculated in units of milliliters per 100 milliliters per minute, $\mathrm{CBV}$ in percentage of total blood volume, and TTP in units of seconds. At our institution, parametric color maps of CBF, CBV, and TTP were generated during the initial emergency department evaluation mainly to increase the accuracy of AIS detection ${ }^{4}$ in the hyperacute period when NCCT may be normal or demonstrate only subtle changes. The "tumor perfusion" mode calculates microvascular permeability and fractional blood volume based on the Patlak method. ${ }^{12}$ PS was calculated in units of milliliters per 100 milliliters per minute. PS color maps were not generated during the initial emergency department evaluation and, therefore, played no role in the decision to administer rtPA or subsequent clinical management.

The Patlak method is a unidirectional 2-compartment model that calculates PS via linear regression. A voxel of brain tissue contains the intracellular, intravascular (plasma), and extravascular (interstitial) spaces. Because a hydrophilic iodinated contrast molecule (tracer) does not cross the hydrophobic cell membrane, contrast enhancement of the intracellular space can be ignored, and only 2 compartments are considered. Below is the well-known formula for the Patlak plot:

$$
C(t)=P S * \int_{0}^{t} b(t) d t+B V * b(t) \rightarrow \frac{C(t)}{b(t)}=P S * \frac{\int_{0}^{t} b(t) d t}{b(t)}+B V
$$

$C(t)$ represents the dynamic enhancement of a tissue voxel after the peripheral injection of contrast material: $C(t)=H U_{\text {brain }}(t)-$ $H U_{\text {native brain }}$, and $b(t)$ similarly represents the dynamic enhancement of a reference blood vessel: $b(t)=H U_{\text {blood }}(t)-H U_{\text {blood native }}$.

The integral of $b(t)$ represents the amount of tracer in blood up to time $t$. Because a tissue voxel contains both the local intravascular and extravascular spaces, $C(t)$ represents the enhancement and thus the amount of tracer within both compartments in the voxel up to time $t$. The second equation above is in the form of a straight line, and a Patlak value $(x, y)$ can be defined for every voxel:

$$
(x, y)=\left(\begin{array}{ll}
\int_{0}^{t} b(t)(d t) \\
b(t) d t
\end{array}, \frac{C(t)}{b(t)}\right)
$$

Because both $x$ and $y$ can be calculated from dynamic data, PS is then interpreted as the slope of the best-fit linear regression line of Patlak values. Measuring enhancement of a small cerebral artery is known to be limited by partial volume effects, and thus the larger caliber superior sagittal sinus is traditionally used as the reference vessel. The main assumption of the Patlak method is negligible reflux of the tracer from the extravascular into the intravascular space. ${ }^{15}$ In our use 


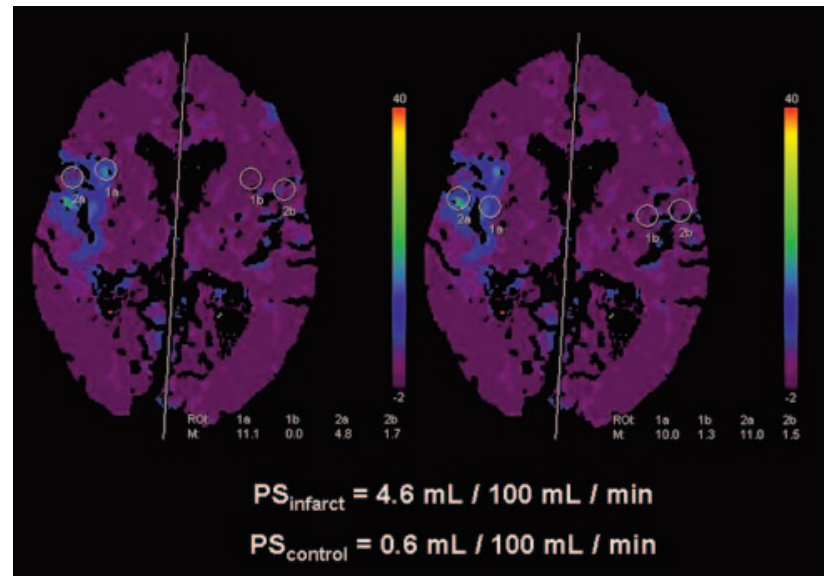

Fig 1. A map of microvascular PS acquired from first-pass dynamic contrast-enhanced images over 60 seconds. There was a region of PS elevation encompassing the right insula cortex and portions of the right frontal and temporal lobes. CBF, CBV, and TTP maps showed hypoperfusion in these regions (not shown) in this 86-year-old woman with an acute right MCA stroke. Four 10-mm circular regions of interest were placed over the region of PS elevation, as depicted here, to acquire 4 PS measurements that were then averaged to give a single value for statistical analysis ( $\left.\mathrm{PS}_{\text {infarct }}\right)$. Mirror regions of interest were automatically placed on the contralateral, nonischemic, homologous hemisphere to provide a control value $\left(\mathrm{PS}_{\text {control }}\right)$. Note that the PS values calculated by the application are scaled to $0.5 \mathrm{~mL} / 100 \mathrm{~mL}$ per minute. No HT was found on follow-up imaging.

of the Syngo Neuro PCT application for this study, the PS for each voxel is calculated over the 60 seconds of the PCT examination.

\section{Data Analyses}

Quantitative data of PS were acquired in the following manner. Four 10-mm-diameter circular regions of interest were manually placed by 1 reader (K.L. with $\sim 2$ years of experience in PCT data analysis) on the PS color map to cover the entire area of a focal PS abnormality or, if the area of abnormality was large, at the central portion of the area of abnormality. Careful attention was paid to the placement of the region of interest as to avoid CSF spaces. Four PS values were acquired from the side of pathology, and the mean value $\left(\mathrm{PS}_{\text {infarct }}\right)$ was used for statistical analysis. Elevated PS was often seen in anatomic locations normally lacking a BBB, such as the choroid plexi in the lateral ventricles. Such locations were always unambiguously recognized and excluded from the analysis. If, and only if, the PS map showed no focal abnormality, the standard perfusion maps were unblinded to guide the placement of 4 regions of interest on the location on the PS map corresponding with the area of severe hypoperfusion/infarction on the perfusion maps. The CBV map was preferentially used, because it has been shown to most specifically correspond with the "infarct core." ${ }^{\prime 9}{ }^{10}$ For every manually placed region of interest, an automated identical region of interest was "mirrored" on the homologous, nonischemic, contralateral location. The mean of these 4 contralateral regions of interest $\left(\mathrm{PS}_{\text {control }}\right)$ served as a control in the statistical analysis. Using 4 circular regions of interest to measure a region of perfusion abnormality has been shown previously to provide the highest intraobserver and interobserver reproducibility. ${ }^{26}$ See Fig 1 for an example of how regions of interest were taken on the PS map. The quantitative analysis of each PS color map was performed while blinded to the standard perfusion maps (unless no focal PS abnormality was seen, as discussed above), the follow-up imaging results, and whether rtPA was given. The reader only knew the patient's side of symptoms (if there was a particular laterality).

Hospital records were reviewed retrospectively to determine each patient's sex, age, presenting neurologic complaint, exact times of all
CT and MR imaging, whether rtPA was administered emergently, and any new or worsening clinical condition during the hospital course. All of the follow-up imaging studies were unblinded after the generation of the PS maps and the collection of PS infarct $_{\text {and }} \mathrm{PS}_{\text {control }}$ values. An infarct with subsequent $\mathrm{HT}$ had its $\mathrm{PS}_{\text {infarct }}$ reclassified as $\mathrm{PS}_{\mathrm{HT}}$, whereas an infarct without $\mathrm{HT}$ had its $\mathrm{PS}_{\text {infarct }}$ reclassified as $\mathrm{PS}_{\mathrm{No}-\mathrm{HT}}$.

The presence of HT was retrospectively evaluated by one interpreter (K.S.K.) by examining the region of infarct for hyperattenuation indicative of blood products on the NCCT and/or signal intensity loss indicative of magnetic susceptibility from deoxyhemoglobin or methemoglobin on the axial DWI $B_{0}$ images. ${ }^{27} \mathrm{HT}$ was categorized according to previously published reports from the European-Australasian Acute Stroke Study II. ${ }^{28,29}$ Specifically, hemorrhagic infarction 1 (HI1) was defined as small petechiae along the peripheral margins of the infarct without mass effect; hemorrhagic infarction 2 (HI2) was defined as confluence of the petechiae located centrally within the infarct without mass effect; parenchymal hematoma 1 (PH1) was defined as a hematoma occupying $30 \%$ or less of the infarct with mild mass effect; parenchymal hematoma 2 ( $\mathrm{PH} 2)$ was defined as a hematoma occupying more than $30 \%$ of the infarct with substantial mass effect.

\section{Statistical Analyses}

$\mathrm{PS}_{\text {infarct }}$ was compared with $\mathrm{PS}_{\text {control }}$ using an exact Wilcoxon pairedsample signed-rank test with an exact 2-sided $P<.05$ being statistically significant. $\mathrm{PS}_{\mathrm{HT}}$ was compared with $\mathrm{PS}_{\mathrm{No}-\mathrm{HT}}$ as a single cohort by using an exact Mann-Whitney test with an exact 2-sided $P<.05$ being statistically significant. This procedure was repeated for each of the 2 subgroups after the cohort was divided according to whether emergent rtPA treatment was given. A receiver operating characteristic (ROC) curve for $\mathrm{PS}_{\mathrm{HT}}$ was calculated for the cohort and for each subgroup, featuring 1-specificity on the $\mathrm{x}$-axis and sensitivity on the $y$-axis for different threshold criteria. The area under each ROC curve (AUC) was determined as a measure of performance, with the maximum possible area being 1.00. The statistical computations were carried out using SAS 9.0 (SAS Institute, Cary, NC), whereas the graphs were generated using Minitab 13.2 (Minitab, State College, Pa).

\section{Results}

Follow-up imaging studies ranged from 6 hours to 15 days after the initial emergency department presentation, with a median of 3 days. All 50 of the AIS patients had at least 1 follow-up study that was performed at least 24 hours after the initial PCT. Of the 50 patients, 39 had both a follow-up NCCT and MR imaging, whereas 11 had follow-up NCCT alone. Of the 50 infarcts, 46 (92\%) were in the middle cerebral artery territory, and $4(8 \%)$ were in the posterior cerebral artery territory. Eighteen patients received rtPA emergently (16 IV and 2 IA). Of these 18 patients, 3 (17\%; all intravenous) developed HT on follow-up imaging: 2 with PH1 and 1 with HI2. Of the 32 patients who did not receive rtPA, 3 (9.2\%) developed HT: 1 each with $\mathrm{PH} 1, \mathrm{HI} 2$, and HI1. The 3 patients with $\mathrm{PH} 1$ all had acute "deterioration of mental status" before the first follow-up imaging examination that detected HT (and thus the clinical deteriorations were attributed to the new hemorrhages). The 3 patients with HI did not have any reported worsening of clinical condition. All of the HTs were found 24-72 hours postictus.

Forty-four (88\%) of the 50 PS maps demonstrated a focal abnormality localized to the region of hypoperfusion. Six PS 


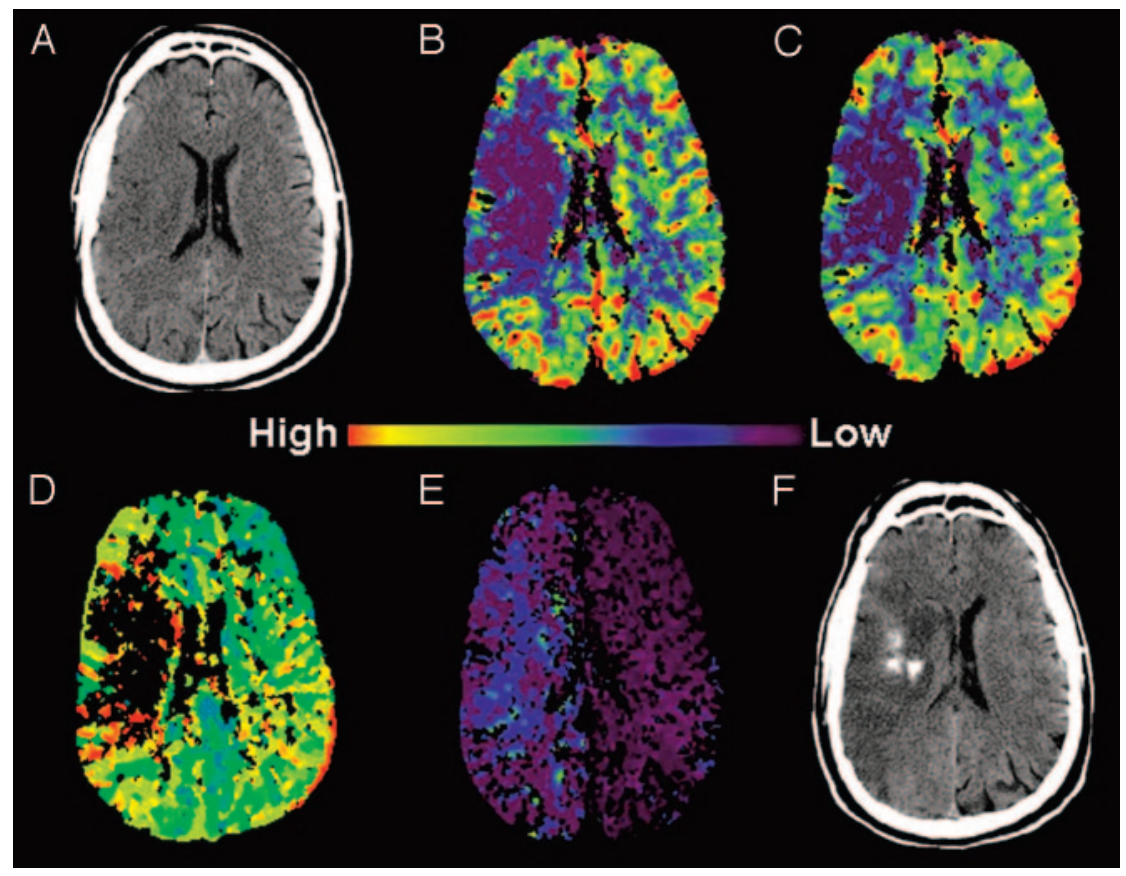

Fig 2. A 53-year-old man with complaint of acute left hemiparesis, presented within 3 hours of symptom onset.

$A$, Initial NCCT showed subtle hypoattenuation in the right frontal lobe with loss of gray-white distinction suspicious for AIS

$B, C$, and $D, C B F, C B V$, and TTP color maps, respectively, showed hypoperfusion in the right basal ganglia and frontal lobe consistent with right MCA AIS.

E, PS color map, from the same raw data used to create the perfusion maps, showed $P_{\text {infarct }}$ of $8.1 \mathrm{~mL} / 100 \mathrm{~mL}$ per minute. Emergent intravenous rtPA was given.

F, NCCT 27 hours after initial presentation revealed frank hemorrhage in the region of infarction ( $\mathrm{PH} 1)$. The patient developed acute deterioration of his mental status 1 hour before this CT.

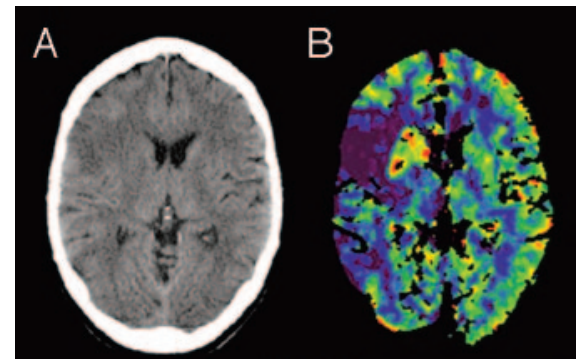

High

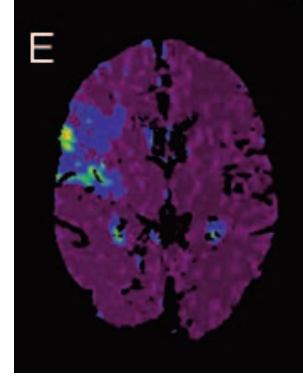

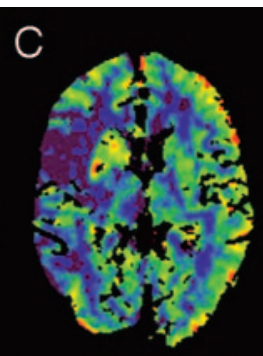

Low
D

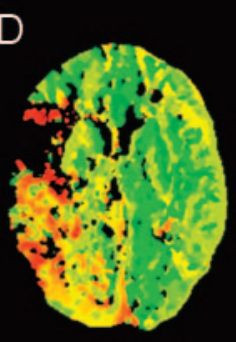

$\mathrm{H}$

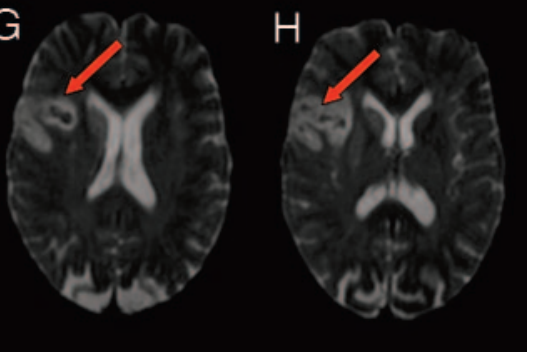

Fig 3. A 68-year-old woman with acute mental status changes, presented within 3 hours of symptom onset.

$A$, Initial NCCT showed loss of gray-white distinction in the right insula (insula ribbon sign) suspicious for AIS

$B, C$, and $D, C B F, C B V$, and TTP color maps, respectively, showed focal perfusion abnormality in the right insula and frontal lobe consistent with acute right MCA AIS.

E, PS color map, from the same raw data used to create the perfusion maps, showed $\mathrm{PS}_{\text {infarct }}$ of $13 \mathrm{~mL} / 100 \mathrm{~mL}$ per minute. No thrombolytic agent was given.

F, NCCT 26 hours after presentation revealed a subtle focus of hyperattenuation in the infarct area.

$G$ and $H, B_{0}$ images from DWI 30 hours after presentation demonstrated foci of signal intensity loss consistent with blood products (HI2).

$\mathrm{PS}_{\mathrm{No}-\mathrm{HT}}$, which ranged from 0 to $5.9 \mathrm{~mL} /$ $100 \mathrm{~mL}$ per minute with a mean $\pm \mathrm{SE}$ of $2.7 \pm 2.0(P<.0001)$. Refer to Figs 2 and 3 for the initial NCCT, CBF, CBV, TTP, PS color maps, and follow-up imaging of 2 of these 6 patients.

maps (12\%) had no focal permeability defect. In the 6 cases of HT, the hemorrhage was localized unambiguously to the region of elevated permeability. Furthermore, in all of the infarcts that developed HT, the region of abnormal PS was localized to the region of very low $\mathrm{CBV}$, probably representing the infarct core. In 3 of the 6 cases of HT, some extension of the periphery of the PS abnormality (no more than $\sim 10 \%$ of the total area) was seen in regions of normal CBV, but low CBF and high TTP were seen, probably representing penumbra. No PS abnormality was seen to extend into normal brain parenchyma.

As a single cohort group, $\mathrm{PS}_{\text {infarct }}$ ranged from 0 to $13 \mathrm{~mL} /$ $100 \mathrm{~mL}$ per minute with a mean $\pm \mathrm{SE}$ of $3.5 \pm 3.1$. This was significantly different from $\mathrm{PS}_{\text {control }}$, which ranged from 0 to $0.8 \mathrm{~mL} / 100 \mathrm{~mL}$ per minute with a mean $\pm \mathrm{SE}$ of $0.28 \pm 0.27$ $(P<.0001)$. All of the $\mathrm{PS}_{\mathrm{HT}}$ ranged from 5.2 to $13 \mathrm{~mL} / 100 \mathrm{~mL}$ per minute with a mean \pm SE of $9.8 \pm 2.9$ compared with
We also stratified the cohort into 2 subgroups according to whether emergent rtPA treatment was given. In the rtPAtreated subgroup, $\mathrm{PS}_{\mathrm{HT}}$ ranged from 5.2 to $13 \mathrm{~mL} / 100 \mathrm{~mL}$ per minute with a mean \pm SE of $8.7 \pm 3.7$. This was significantly different from the $\mathrm{PS}_{\mathrm{No}-\mathrm{HT}}$ within this subgroup, which ranged from 0 to $5.9 \mathrm{~mL} / 100 \mathrm{~mL}$ per minute with a mean $\pm \mathrm{SE}$ of $3.1 \pm 2.1(P=.0172)$. In the subgroup not treated with rtPA, $\mathrm{PS}_{\mathrm{HT}}$ ranged from 9.8 to $13 \mathrm{~mL} / 100 \mathrm{~mL}$ per minute with a mean \pm SE of $11 \pm 1.5$. This was also significantly different from the $\mathrm{PS}_{\mathrm{No}-\mathrm{HT}}$ within this subgroup, which ranged from 0 to $6.0 \mathrm{~mL} / 100 \mathrm{~mL}$ per minute with a mean $\pm \mathrm{SE}$ of $2.5 \pm 1.9$ $(P=.0004)$. All 50 of the $\mathrm{PS}_{\text {infarct }}$ values are shown in a scatterplot in Fig 4.

For the non-rtPA-treated subgroup, there was complete separation of $\mathrm{PS}_{\mathrm{HT}}$ and $\mathrm{PS}_{\mathrm{No}-\mathrm{HT}}$ measurements; any $\mathrm{PS}_{\text {infarct }}$ between 6.0 and $9.8 \mathrm{~mL} / 100 \mathrm{~mL}$ per minute can be used as a threshold value to predict HT with a sensitivity and specificity 


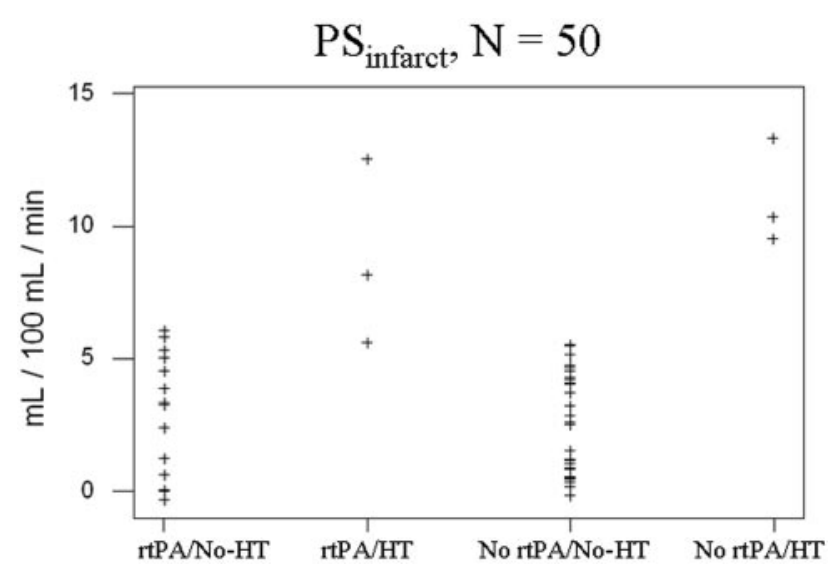

Fig 4. Scatterplot of the $50 \mathrm{PS}_{\text {infarct }}$ values, categorized by whether emergent rtPA was given and whether subsequent HT occurred. Note the separation of $\mathrm{PS}_{\mathrm{HT}}$ values (second and fourth columns) from those of $\mathrm{PS}_{\mathrm{No}-\mathrm{HT}}$ (first and third columns).

of $100 \%$. Consequently, the data in the non-rtPA-treated subgroup yielded an ROC curve with AUC of 1.00 (curve not shown). For the rtPA-treated subgroup, threshold $\mathrm{PS}_{\text {infarct }}$ criteria of 4.99 and $5.88 \mathrm{~mL} / 100 \mathrm{~mL}$ per minute with a sensitivity and specificity of $100 \%$ and $80 \%$ and $66.7 \%$ and $100 \%$, respectively, generated an ROC curve with AUC of 0.933 . Taking the entire cohort as a single group, threshold PS $_{\text {infarct }}$ criteria of 5.16 and $5.99 \mathrm{~mL} / 100 \mathrm{~mL}$ per minute with a sensitivity and specificity of $100 \%$ and $88.6 \%$ and $83.3 \%$ and $100 \%$, respectively, generated an ROC curve with AUC of 0.981. These curves are shown in Fig 5.

\section{Discussion}

The purpose of our study was to determine whether elevated microvascular permeability can be detected in very early strokes by using first-pass dynamic contrast-enhanced images from PCT and, if so, whether PS infarct $_{\text {values were significantly }}$ higher for those infarcts that were destined for HT. We used a retrospective study design in which we measured PS in the region of hypoperfusion and then examined the follow-up imaging data to determine the presence of HT. Our results showed that elevated PS can be detected during this hyperacute period using first-pass dynamic CT data. Despite some overlap between $\mathrm{PS}_{\mathrm{HT}}$ and $\mathrm{PS}_{\mathrm{No}-\mathrm{HT}}$ in the cohort, differences in their mean values were statistically significant. We stratified the cohort into 2 subgroups according to rtPA treatment, and differences in $\mathrm{PS}_{\mathrm{HT}}$ and $\mathrm{PS}_{\mathrm{No}-\mathrm{HT}}$ remained significantly different in both. ROC curves were generated with selected $\mathrm{PS}_{\text {infarct }}$ threshold criteria, and the curves demonstrate excellent discrimination with AUCs ranging from 0.933 (rtPA subgroup) to 1.00 (non-rtPA subgroup).

A defect in permeability was found mostly within the infarct core, which was expected, because this region contains the most severely injured tissue. Although there are many studies that report various clinical and molecular variables predictive of $\mathrm{HT},{ }^{30,31}$ imaging BBB permeability is an intuitive and conceptually attractive method to assess for HT risks. Abnormal BBB permeability can be seen qualitatively as parenchymal enhancement in conventional delayed postgadolinium enhanced MR imaging, with enhancement in the early period of AIS being predictive of HT. ${ }^{32-34}$ A conventional contrast-enhanced CT is not a component of our multimodal stroke series protocol, and no contrast-enhanced CT or MR imaging was performed during the follow-up period for our cohort. Therefore, we could not assess for conventional parenchymal enhancement in a region with elevated PS. Future investigations should attempt to elucidate any association between early qualitative enhancement and quantitatively elevated PS and compare their accuracies in predicting HT.

In this study, patients who received rtPA therapy had a higher risk of HT (17\% versus $9.2 \%$ ), as expected. In addition, patients with high $\mathrm{PS}_{\text {infarct }}$ who then received rtPA tended to develop parenchymal hematomas ( 2 of 3 ) that were coincident with clinical deterioration. Patients with high $\mathrm{PS}_{\text {infarct }}$ who were not treated with rtPA tended to develop smaller petechial hemorrhagic infarctions (HIs; 2 of 3 ) that were asymptomatic. When discovered, HI (even if asymptomatic) may require a closer surveillance of blood pressure and discontinuation of anticoagulant therapy to prevent the risk of a larger hemorrhage. In such a clinical scenario, an elevated PS measurement can still have prospective value by guiding subsequent in-hospital management in patients for whom the decision has already been made to not treat with $\mathrm{rtPA}$.

Some authors have argued that asymptomatic petechial HI can be a favorable prognostic sign, indicating early successful recanalization and reperfusion. ${ }^{35}$ In this cohort, follow-up angiographic data were not obtained in the 6 patients with HT. Thus we cannot determine which, if any, was associated with recanalization that may have been early versus late or spontaneous versus rtPA-induced. These variables should ideally be accounted for in future studies.

The mean $\mathrm{PS}_{\mathrm{HT}}$ was lower in the rtPA treatment group by $\sim 21 \%$ (8.7 versus $11 \mathrm{~mL} / 100 \mathrm{~mL}$ per minute). This finding is compatible with recent molecular studies indicating that rtPA itself may further promote opening of the BBB by activating lipoprotein receptor-related protein (LRP), which increases BBB permeability. ${ }^{36}$ LRP can also upregulate matrix metalloproteinase-9, a mediator of extracellular proteolysis of the neurovascular matrix. ${ }^{37,38}$

In comparison to the observations by Kassner et al, ${ }^{24}$ we examined a larger study population consisting of both patients with and without rtPA treatment. Moreover, this study examined the use of PCT, which has several advantages over perfusion-weighted MR imaging (PWI) in stroke evaluation. Because an NCCT of the brain is ubiquitously the first imaging study obtained for patients with suspected AIS, a PCT performed immediately afterward (while the patient remains on the scanner table) is fast, convenient, inexpensive, and widely available, without the need for specialized equipment or personnel. A perfusion value obtained with PCT is generally regarded as a more accurate hemodynamic measurement over PWI, because the concentration of iodinated contrast agent is linear with $\mathrm{x}$-ray attenuation. ${ }^{15}$ In addition, measurements are unaffected by the tissue microenvironment, which may be problematic in PWI. The major limitation of PCT is its current inability to provide quantitative whole-brain imaging, though this problem will probably be obviated in the near future with the next generation of multidetector scanners.

The limitations of the study are its retrospective design and a number of potential biases. We did not use multiple readers for the quantitative analysis, and, as such, we cannot assess for interobserver variability in PS measurements. In addition, our 

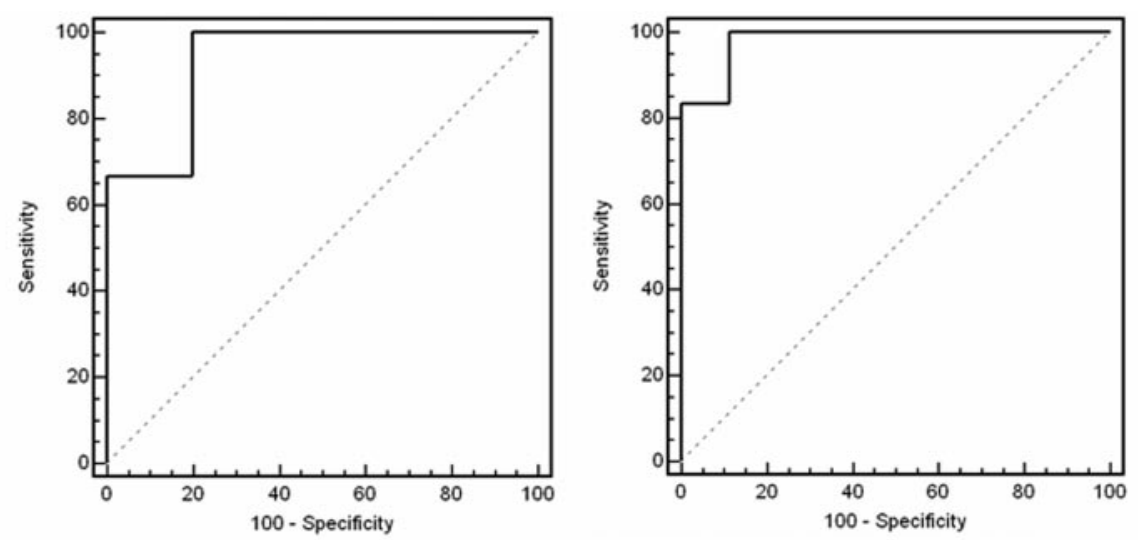

Fig 5. ROC curves for the rtPA-treated subgroup (left) and the entire cohort (right) with the table

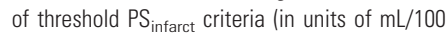
$\mathrm{mL}$ per minute) used to generate the curves. For the rtPA-treated subgroup and the entire cohort the AUC was 0.933 and 0.981 , respectively. The data in the non-rtPA-treated subgroup produced an ROC curve with AUC of 1.00 (data not shown), because there was complete separation of $\mathrm{PS}_{\mathrm{HT}}$ and $\mathrm{PS}_{\mathrm{No}-\mathrm{HT}}$ (Fig 4).

\begin{tabular}{|c|c|c|c|c|c|}
\hline \multicolumn{3}{|c|}{ rtPA, N = 18 } & \multicolumn{3}{c|}{ All, N = 50 } \\
\hline Threshold & Sensitivity\% & Specificity\% & Threshold & Sensitivity\% & Specificity\% \\
\hline$>4.9875$ & $100.0(3 / 3)$ & $80(12 / 15)$ & $>5.1625$ & $100(6 / 6)$ & $88.6(39 / 44)$ \\
\hline$>5.2125$ & $66.7(2 / 3)$ & $80(12 / 15)$ & $>5.2125$ & $83.3(5 / 6)$ & $88.6(39 / 44)$ \\
\hline$>5.875$ & $66.7(2 / 3)$ & $100(15 / 15)$ & $>5.9875$ & $83.3(5 / 6)$ & $100(44 / 44)$ \\
\hline
\end{tabular}

cohort is only a small percentage of all of the AIS patients who presented to the emergency department during the study period. The reality is that most patients present much later to the emergency department, when their infarcts may be more extensive and complete. The association between $\mathrm{PS}_{\text {infarct }}$ and HT in this larger subpopulation is not answered by this study.

The Patlak method, a unidirectional 2-compartment model, is the simplest approximation method for calculating permeability. ${ }^{15}$ The Patlak method assumption that contrast does not diffuse back into the intravascular space is appropriate when dealing with first-pass dynamic data. During the first pass of contrast through a region with a BBB defect, a small amount of extravascular leakage of tracer occurs, ${ }^{19,39}$ though most will remain in the intravascular space (allowing for perfusion calculations). In tumor imaging, first-pass permeability is measured with a high scan frequency $2^{*}$ susceptibility technique. ${ }^{18,19}$ Some investigators may argue that steady-state data analyzed with a bidirectional model should be more accurate in calculating permeability. ${ }^{15}$ Although this has been the general viewpoint in tumor imaging, we are reminded of the recent observations by Cha et $\mathrm{al}^{19}$ that, for gliomas, volume transfer coefficient values derived from a first-pass $\mathrm{T} 2{ }^{\star}$ technique in less than 1.5 minutes show a linear correlation with those derived from a steady-state T1 technique over 6 minutes. For stroke imaging, there is no study to our knowledge that compares the accuracy of permeability generated from unidirectional modeling of first-pass data against bidirectional modeling of steady-state data. Clearly, measuring PS under steady-state conditions requires methodologies different from those described in this study. Further investigations will be needed to compare different acquisition protocols.

\section{Conclusions}

Elevated microvascular permeability was detectable in patients with very early AIS by using dynamic data acquired from first-pass contrast-enhanced PCT. The permeability of infarcts that resulted in HT was significantly higher, irrespective of rtPA treatment. The use of this information in emergent stroke management and, in particular, its effect on rates of rtPA eligibility will require further, prospective investigations.

\section{Acknowledgments}

We thank Dr Ernst Klotz for useful discussions and assistance in the preparation of this article.

\section{References}

1. Axel L. Cerebral blood flow determination by rapid-sequence computed tomography. Radiology 1980;137:679-86

2. Koenig M, Klotz E, Luka B, et al. Perfusion CT of the brain: diagnostic approach for early detection of ischemic stroke. Radiology 1998;209:85-93

3. Klotz E, König M. Perfusion measurements of the brain: using dynamic CT for the quantitative assessment of cerebral ischemia in acute stroke. Eur J Radiol 1999;30:170-84

4. Mayer TE, Hamann GF, Baranczyk J, et al. Dynamic CT perfusion imaging of acute stroke. AJNR Am J Neuroradiol 2000;21:1441-49

5. Rother J, Jonetz-Mentzel L, Fiala A, et al. Hemodynamic assessment of acute stroke using dynamic single-slice computed tomographic perfusion imaging. Arch Neurol 2000;57:1161-66

6. Nabavi DG, Cenic A, Henderson S, et al. Perfusion mapping using computed tomography allows accurate prediction of cerebral infarction in experimental brain ischemia. Stroke 2001;32:175-83

7. Eastwood JD, Lev MH, Azhari T, et al. CT perfusion scanning with deconvolution analysis: pilot study in patients with acute middle cerebral artery stroke. Radiology 2002;222:227-36

8. Eastwood JD, Lev MH, Wintermark M, et al. Correlation of early dynamic CT perfusion imaging with whole-brain MR diffusion and perfusion imaging in acute hemispheric stroke. AJNR Am J Neuroradiol 2003;24:1869-75

9. Schaefer PW, Roccatagliata L, Ledezma C, et al. First-pass quantitative CT perfusion identifies thresholds for salvageable penumbra in acute stroke patients treated with intra-arterial therapy. AJNR Am J Neuroradiol 2006;27:20-25

10. Wintermark M, Flanders AE, Velthuis B, et al. Perfusion-CT assessment of infarct core and penumbra: receiver operating characteristic curve analysis in 130 patients suspected of acute hemispheric stroke. Stroke 2006;37:979-85

11. Lyden PD, Zivin JA. Hemorrhagic transformation after cerebral ischemia: mechanisms and incidence. Cerebrovasc Brain Metab Rev 1993;5:1-16

12. Patlak CS, Blasberg RG, Fenstermacher JD. Graphical evaluation of blood-tobrain transfer constants from multiple-time uptake data. J Cereb Blood Flow Metab 1983;3:1-7

13. Tofts PS. Modeling tracer kinetics in dynamic Gd-DTPA MR imaging. J Magn Reson Imaging 1997;7:91-101

14. Tofts PS, Brix G, Buckley DL, et al. Estimating kinetic parameters from dy- 
namic contrast-enhanced T(1)-weighted MRI of a diffusable tracer: standardized quantities and symbols. J Magn Reson Imaging 1999;10:223-32

15. Lee TY, Purdie TG, Stewart E. CT imaging of angiogenesis. Q J Nucl Med 2003;47:171-87

16. Roberts HC, Roberts TP, Brasch RC, et al. Quantitative measurement of microvascular permeability in human brain tumors achieved using dynamic contrast-enhanced MR imaging: correlation with histologic grade. AJNR Am J Neuroradiol 2000;21:891-99

17. Yang S, Law M, Zagzag D, et al. Dynamic contrast-enhanced perfusion MR imaging measurements of endothelial permeability: differentiation between atypical and typical meningiomas. AJNR Am J Neuroradiol 2003;24:1554-59

18. Law M, Yang S, Babb JS, et al. Comparison of cerebral blood volume and vascular permeability from dynamic susceptibility contrast-enhanced perfusion MR imaging with glioma grade. AJNR Am J Neuroradiol 2004;25:746-55

19. Cha S, Yang L, Johnson G, et al. Comparison of microvascular permeability measurements, $\mathrm{K}$ (trans), determined with conventional steady-state T1weighted and first-pass $\mathrm{T}^{\star}{ }^{*}$-weighted $\mathrm{MR}$ imaging methods in gliomas and meningiomas. AJNR Am J Neuroradiol 2006;27:409-17

20. Kuroiwa T, Ting $\mathrm{P}$, Martinez $\mathrm{H}$, et al. The biphasic opening of the blood-brainbarrier to proteins following temporary middle cerebral artery occlusion. Acta Neuropathol 1985;68:122-29

21. Pluta R, Lossinsky AS, Wisniewski HM, et al. Early blood-brain barrier changes in the rat following transient complete cerebral ischemia induced by cardiac arrest. Brain Res 1994;633:41-52

22. Belayev L, Busto R, Zhao W, et al. Quantitative evaluation of blood-brain barrier permeability following middle cerebral artery occlusion in rats. Brain Res 1996;739:88-96

23. Latour LL, Kang DW, Ezzeddine MA, et al. Early blood-brain barrier disruption in human focal brain ischemia. Ann Neurol 2004;56:468-77

24. Kassner A, Roberts T, Taylor K, et al. Prediction of hemorrhage in acute ischemic stroke using permeability MR imaging. AJNR Am J Neuroradiol 2005;26:2213-17

25. Rowley HA. Extending the time window for thrombolysis: evidence from acute stroke trials. Neuroimaging Clin N Am 2005;15:575-87

26. Wetzel SG, Cha S, Johnson G, et al. Relative cerebral blood volume measurements in intracranial mass lesions: interobserver and intraobserver reproducibility study. Radiology 2002;224:797-803

27. Lam WWM, So NMC, Wong KS, et al. B0 images obtained from diffusion- weighted echo planar sequences for the detection of intracerebral bleeds. J Neuroimaging 2003;13:99-105

28. Larrue V, von Kummer RR, Muller A, et al. Risk factors for severe hemorrhagic transformation in ischemic stroke patients treated with recombinant tissue plasminogen activator: a secondary analysis of the European-Australasian Acute Stroke Study (ECASS II). Stroke 2001;32:438-41

29. Trouillas P, von Kummer R. Classification and pathogenesis of cerebral hemorrhages after thrombolysis in ischemic stroke. Stroke 2006;37:556-61

30. Kidwell CS, Saver JL, Carneado J, et al. Predictors of hemorrhagic transformation in patients receiving intra-arterial thrombolysis. Stroke 2002;33:717-24

31. Wang X, Tsuji K, Lee SR, et al. Mechanisms of hemorrhagic transformation after tissue plasminogen activator reperfusion therapy for ischemic stroke. Stroke 2004;35(suppl 1):2726-30

32. Knight RA, Barker PB, Fagan SC, et al. Prediction of impending hemorrhagic transformation in ischemic stroke using magnetic resonance imaging in rats. Stroke 1998;29:144-51

33. Neumann-Haefelin C, Brinker G, Uhlenkuken U, et al. Prediction of hemorrhagic transformation after thrombolytic therapy of clot embolism: an MRI investigation in rat brain. Stroke 2002;33:1392-98

34. Vo KD, Santiago F, Lin W, et al. MR imaging enhancement patterns as predictors of hemorrhagic transformation in acute ischemic stroke. AJNR Am J Neuroradiol 2003;24:674-79

35. Molina CA, Alvarez-Sabin J, Montaner J, et al. Thrombolysis-related hemorrhagic infarction: a marker of early reperfusion, reduced infarct size, and improved outcome in patients with proximal middle cerebral artery occlusion. Stroke 2002;33:1551-56

36. Yepes M, Sandkvist M, Moore EG, et al. Tissue-type plasminogen activator induces opening of the blood-brain barrier via the LDL receptor-related protein. J Clin Invest 2003;112:1533-40

37. Wang X, Lee SR, Arai K, et al. Lipoprotein receptor-mediated induction of matrix metalloproteinase by tissue plasminogen activator. Nat $\mathrm{Med}$ 2003;9:1313-17

38. Tsuji K, Aoki T, Tejima E, et al. Tissue plasminogen activator promotes matrix metalloproteinase-9 upregulation after focal cerebral ischemia. Stroke 2005;36:1954-59

39. Hackländer T, Reichenbach JR, Mödder U. Comparison of cerebral blood volume measurements using the $\mathrm{T} 1$ and $\mathrm{T} 2{ }^{\star}$ methods in normal human brains and brain tumors. J Comput Assist Tomogr 1997;21:857-66 